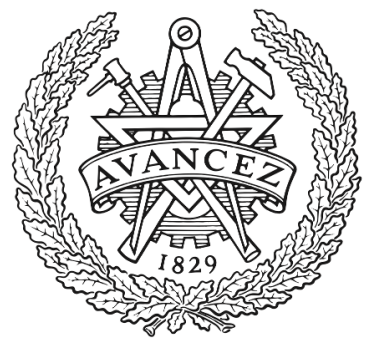

CHALMERS

UNIVERSITY OF TECHNOLOGY

\title{
Impact of Stripe Shape on the Reflectivity of Monolithic High Contrast Gratings
}

Downloaded from: https://research.chalmers.se, 2023-04-26 11:55 UTC

Citation for the original published paper (version of record):

Marciniak, M., Chang, T., Lu, T. et al (2021). Impact of Stripe Shape on the Reflectivity of

Monolithic High Contrast Gratings. ACS Photonics, 8(11): 3173-3184.

http://dx.doi.org/10.1021/acsphotonics.1c00850

N.B. When citing this work, cite the original published paper. 


\section{Impact of Stripe Shape on the Reflectivity of Monolithic High Contrast Gratings}

Magdalena Marciniak, Tsung-Scheng Chang, Tien-Chang Lu, Filip Hjort, Åsa Haglund, Łucja Marona, Matusz Gramala, Paweł Modrzyński, Robert Kudrawiec, Krzysztof Sawicki, Rafał Bożek, Wojciech Pacuski, Jan Suffczyński, Marcin Gębski, Artur Broda, Jan Muszalski, James A. Lott, and Tomasz Czyszanowski*

Cite This: ACS Photonics 2021, 8, 3173-3184

Read Online

ACCESS | Lill Metrics \& More | 期 Article Recommendations

Supporting Information

ABSTRACT: Monolithic high contrast gratings (MHCGs) composed of a one-dimensional grating patterned in a monolithic layer provide up to $100 \%$ optical power reflectance and can be fabricated in almost any semiconductor and dielectric material used in modern optoelectronics. MHCGs enable monolithic integration, polarization selectivity, and versatile phase tuning. They can be from 10 to 20 times thinner than distributed Bragg reflectors. The subwavelength dimensions of MHCGs significantly reduce the possibility of ensuring the smoothness of the sidewalls of the MHCG stripes and make precise control of the shape of the MHCG stripe cross-section difficult during the etching process. The question is then whether it is more beneficial to improve the etching methods to obtain a perfect cross-section shape, as assumed by the design, or whether it is possible to find geometrical
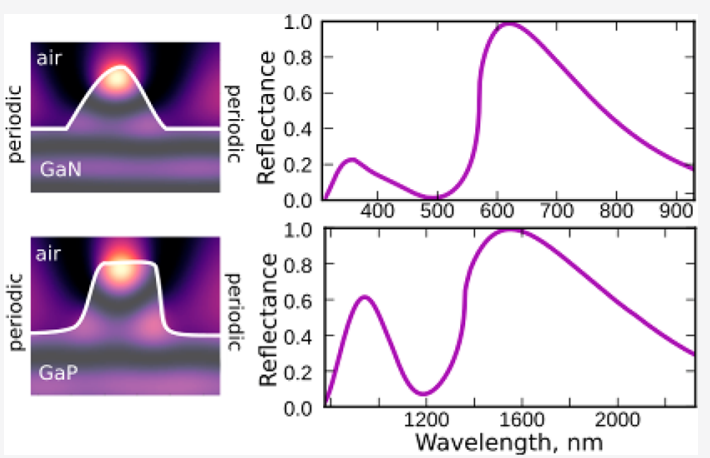
parameters that enable high optical power reflectance using the shape that a given etching method provides. Here, we present a numerical study supported by the experimental characterization of MHCGs fabricated in various materials using a variety of common surface nanometer-scale shaping methods. We demonstrate that MHCG stripes with an arbitrary cross-section shape can provide optical power reflectance of nearly $100 \%$, which greatly relaxes their fabrication requirements. Moreover, we show that optical power reflectance exceeding $99 \%$ with a record spectral bandwidth of more than $20 \%$ can be achieved for quasi-trapezoidal cross-sections of MHCGs. We also show that sidewall corrugations of the MHCG stripes have only a slight impact on MHCG optical power reflectance if the amplitude of the corrugation is less than $16 \%$ of the MHCG period. This level of stripe fabrication precision can be achieved using the most current surface etching methods. Our results are significant for the design and production of a variety of photonic devices employing MHCGs. The flexibility with regard to crosssection shape facilitates the reliable fabrication of highly reflective subwavelength grating mirrors. This in turn will enable the manufacture of monolithically integrated high-quality-factor optical micro- and nanocavity devices.

KEYWORDS: monolithic high contrast grating, subwavelength grating, optical power reflectance

$\mathrm{H}$ igh contrast gratings (HCGs) are composed of high refractive index material embedded in or implemented on a low refractive index material. ${ }^{1,2}$ Monolithic high contrast gratings (MHCGs) are a special case of HCGs, where the refractive index of the grating is the same as that of the layer beneath the grating. An MHCG therefore constitutes a simple one-dimensional grating patterned in a monolithic bulk layer. ${ }^{3}$ MHCGs enable arbitrarily high power reflectance when the grating has a refractive index larger than 1.75 . $^{4}$ They facilitate strong polarization discrimination and phase tuning ${ }^{5}$ of reflected and transmitted light, even when the thickness of the grating is as little as half of the wavelength in the grating material. In ref 6, an MHCG used as the top mirror of a vertical resonant cavity in a polariton laser enabled a high quality factor ( $Q$-factor) cavity and efficient optical pumping through the MHCG. In ref 7, an electrically injected MHCG- based vertical-cavity surface-emitting laser (VCSEL) emitting at $980 \mathrm{~nm}$ was demonstrated with $30 \%$ lower epitaxial structure height than a conventional VCSEL and strong discrimination of the lateral modes. Since the properties of MHCGs are to a large extent independent of the material in which the MHCGs are patterned, their applications are not limited to the replacement of DBR mirrors in VCSELs, as noted in refs 6 and 7.

Received: June 10, 2021

Published: October 28, 2021 

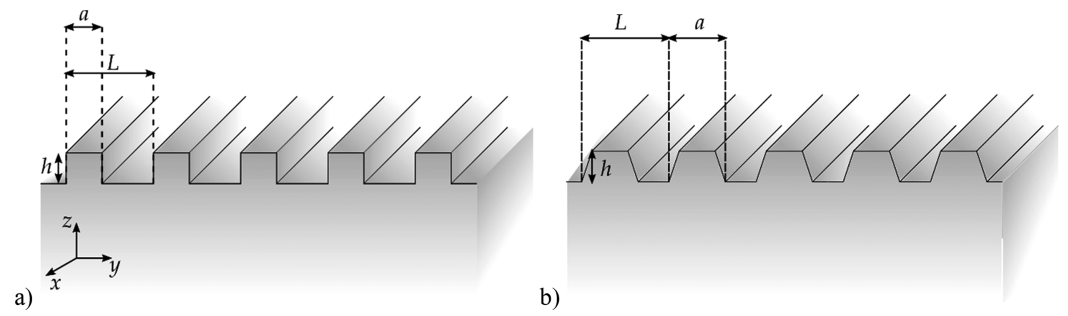

Figure 1. Schematic structure of monolithic high contrast gratings (MHCGs) with a (a) rectangular and (b) trapezoidal cross-section of the stripes: $L$, period of the grating; $h$, height of the stripes; $F$, fill factor, as the ratio of the width of the stripe $a$ to the grating period $L$. The coordinate system used in the paper is indicated.

MHCGs open a new route to the facile design and fabrication of highly reflecting mirrors ${ }^{8}$ and high $Q$-factor optical microcavities. ${ }^{9}$ The enhanced interaction of light and matter in such cavities has found many applications in science and technology. Numerous contemporary physics experiments use optical cavities to enhance detection sensitivity, ${ }^{10}$ nonlinear interactions, ${ }^{11}$ single-photon generation, ${ }^{12}$ coupling of distant quantum emitters, ${ }^{13}$ and Bose-Einstein condensation of exciton-polaritons ${ }^{14}$ and photons. ${ }^{15}$ Important aspects in the design of optical cavities include the arbitrariness of the material from which they can be made, the ability to tune the Q-factor of the cavity, and the possibility of engineering the cavity dispersion. All these features are facilitated by one or more MHCG cavity mirrors, ${ }^{9}$ which enables tuning of the resonant wavelength and $Q$-factor in the case of cavities and of the reflected phase in the case of mirrors, simply by modifying the grating dimensions.

Although the fabrication of MHCGs relies on wellestablished lithographic and etching methods, which enable control of the grating period, the subwavelength dimensions of MHCGs significantly reduce the possibility of ensuring the smoothness of the sidewalls of the MHCG stripes and make precise control of the shape of the MHCG stripe cross-section (CS) difficult. With such small dimensions, MHCG processing becomes strongly dependent on the semiconductor material of the grating and the lithographic and etching methods used. $^{16-18}$ Typically, the fabrication of an MHCG mirror requires a two-step process: (1) transferring the pattern to the sample surface; (2) etching the pattern into the sample. The first step can be performed by photolithography, electron beam lithography (EBL), or nanoimprint lithography (NIL). EBL is most often used to create subwavelength structures for visible and near-infrared light, as small features can be made with an accuracy of more than $10 \mathrm{~nm} .{ }^{19}$ The resolution of photolithography is limited by the wavelength used usually from UV spectral range and typically required an expansive stepper equipment. The resolution of photolithography is therefore limited by the wavelength used. The resolution of NIL can be in the order of several nanometers. ${ }^{20-22}$ The second processing step may be wet etching, during which the sample is immersed in a chemical etchant, or dry etching, during which the material is removed by the reactive and high-energy ions in an etching plasma. Wet etching is characterized by higher selectivity to the etched material, whereas dry etching provides greater anisotropy and control over etch depth, via inductively coupled plasma reactive ion etching (ICP-RIE). ${ }^{16-18,23}$ Another possible surface etching process is focused ion beam (FIB), which eliminates the transfer processing step. The drawbacks of this one-step method include the difficulty of achieving patterns with sharp edges due to the quasi-Gaussian spatial distribution of the ion beam ${ }^{16-18}$ and the relatively time-consuming process in comparison to the more common two-step methods.

Regardless of the fabrication method used, there are numerous processing parameters that enable tuning of the CS. However, the number of parameters requires nontrivial optimization of the production technology to achieve the desired shape, which is sometimes extremely difficult or even impossible to achieve. Therefore, the question arises of whether it is more beneficial to improve the etching methods to obtain a perfect CS shape, as assumed by the design, or whether it is possible to find geometrical parameters that enable high optical power reflectance using the shape that a given method provides. In this work, we explore the second possibility, which requires a combination of experimental characterization and numerical simulations to achieve the final goal of a high optical power reflectance MHCG. Another issue of concern in this paper is the narrow reflection spectrum of MHCGs as compared to the spectra of DBRs ${ }^{24,25}$ and HCGs. $^{26}$ In ref 4 we showed that a GaAs MHCG with a rectangular CS has a reflectance stopband width comparable to that of a GaAs-based DBR, which is half as narrow as the spectra of an HCG.

Here, we show by simulations supported by experimental characterization that by appropriate adjustment of the grating parameters, such as the etching depth and the grating period, the total power reflectance of an MHCG can be exactly or nearly $100 \%$. This is regardless of its CS, which may be any arbitrary theoretical shape, and particularly any shape fabricated using different surface shaping processes and materials. Before the main analysis, we provide an example in which the reflection spectrum of an MHCG with a trapezoidal CS is experimentally characterized and compared to the numerical model, showing very high agreement between model and experiment. The experimental samples used in the further analysis were intentionally imperfect realizations of MHCGs or even first realization attempts. The shapes were chosen to support the conclusions regarding the arbitrariness of the CS. We also show that a rectangular-shaped CS is not optimal with respect to the width of the reflection stopband and that the spectral width of the reflection stopband can be significantly enhanced, even exceeding the reflection stopband of an HCG, provided the CS is properly tuned. Finally, we investigate the influence on the reflection spectrum of corrugation along the sidewalls of the MHCG stripes, which often occurs during the etching process. 


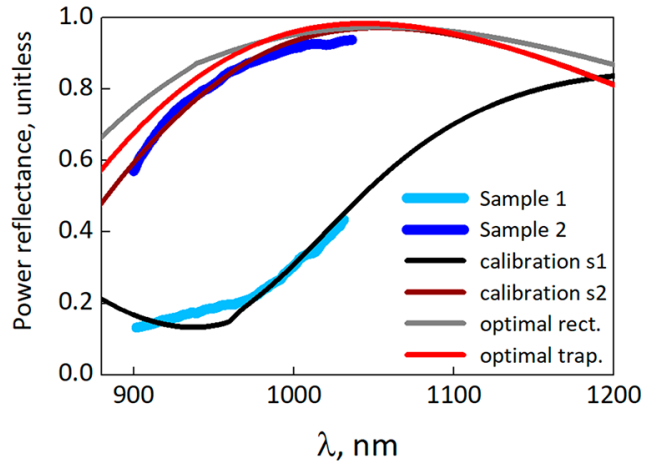

a)
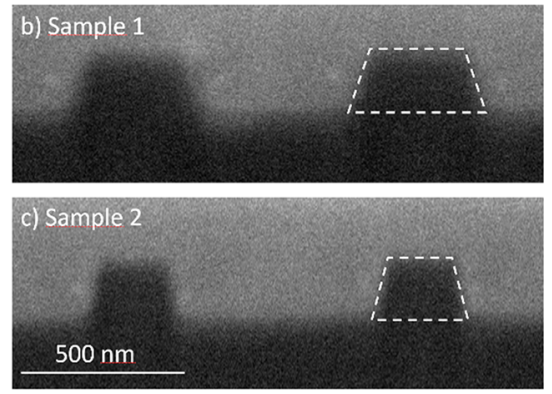

b)

Figure 2. (a) Measured optical power reflectance spectrum for samples 1 (light blue) and 2 (dark blue) for the GaAs monolithic high contrast gratings illuminated by TE polarized light. Black and red lines indicate calculated reflection spectra for samples 1 (calibration s1) and 2 (calibration s2), with parameters calibrated based on experimental characteristics. Gray and red lines represent calculated power reflectance from the MHCG with a rectangular (optimal rect.) or trapezoidal (optimal trap.) CS, respectively, with optimal parameters enabling $97 \%$ power reflectance. (b, c) Cross-sectional SEM images of samples 1 and 2, respectively. The SEM images were made at an angle of $25^{\circ}$ with respect to the plane of the MHCG layer and graphically stretched in the vertical direction to display a realistic aspect ratio.

\section{INFLUENCE OF CROSS-SECTIONAL SHAPE ON THE MAXIMAL POWER REFLECTANCE OF THE MHCG}

The geometric parameters of an MHCG with a rectangularshaped CS are defined graphically in Figure 1a, where $L$ is the period of the grating, $h$ is the height of the stripes (i.e., the etching depth from the surface), and $F$ is the fill factor, defined as the ratio of the width of the stripes $(a)$ to the period of the grating $(L)$. In the case of a nonrectangular CS, the width of the stripe is defined as the width of the stripe base, as shown in Figure $1 \mathrm{~b}$.

In the numerical calculations, we simulate a single period of the MHCG with a grating stripe of infinite length and impose periodic boundary conditions that elongate the periodicity of the grating to infinity. We also assume semi-infinite thickness of air above the MHCG and a semi-infinite layer beneath the grating, made of the same material as the grating. To determine the optical power reflectance of the gratings, we use the plane-wave reflection transformation method (PWRTM). ${ }^{27}$ This method is a fully vectorial optical model that transforms Maxwell's equations into one characteristic equation in which we assume that the structure is composed of parallel layers in the propagation direction. The electromagnetic fields, as well as the distribution of the electrical permittivity, are decomposed into a complete set of exponential functions. We consider two orthogonal polarizations: transverse-electric (TE), for which electric field of electromagnetic wave is parallel to the stripes, and transversemagnetic (TM), where the magnetic component of the electromagnetic field is parallel to the grating stripes. We have validated the accuracy of the method in numerous analyses ${ }^{4,8,28}$ by comparison with experimental reflection spectra of MHCGs and with simulated transmission spectra from other numerical models, ${ }^{29}$ showing very good agreement. These previous studies support the results and conclusions presented here. We determine the optimal parameters of the MHCGs, which are a result of three-dimensional maximization of power reflectance assuming grating parameters $L, F$, and $h$ as variables. We search for the optimal parameters corresponding to local maxima of power reflectance close to $100 \%$ for the smallest $h$.
Let us begin by validating our numerical model based on the experimental reflection spectra of a GaAs MHCG, of which the fabrication process is already well established. ${ }^{30}$ The example also presents a method for optimizing the MHCG, based on adjusting the grating spatial parameters, taking into account the CS of the grating stripes that results from the fabrication method. Our initial intention was to realize an MHCG with a rectangular CS with the parameters $L=800 \mathrm{~nm}, F=0.35$, and $h=180 \mathrm{~nm}$, capable of maximum 97\% reflection at the 1050 $\mathrm{nm}$ wavelength for TE polarization (gray line in Figure 2a). The SEM image in Figure $2 b$ shows sample 1 processed using EBL and ICP-RIE. The parameters are close to those intended $(L=820 \pm 10 \mathrm{~nm}, h=190 \pm 11 \mathrm{~nm})$, but the sample has a trapezoidal CS with a sidewall angle of $16^{\circ}$. The top base of the trapezoid is $290 \pm 10 \mathrm{~nm}$, which corresponds to the designed $F$ $=0.35$. The bottom base is $400 \pm 10 \mathrm{~nm}$, which corresponds to $F=0.48$. In the following analysis of the trapezoidal CS, we define $F$ as corresponding to the bottom base. In the measurement setup, a tunable Ti:sapphire laser was used in the characterization of optical power reflectance, enabling spectral range tuning from 950 to $1050 \mathrm{~nm}$ and nearly normal incidence across the beam with respect to the MHCG surface. More details on the experimental setup and the measurements are provided in ref 8 .

The measured reflectance spectrum of sample 1 (light blue line in Figure 2a) deviates significantly from the predicted spectrum for a rectangular CS (gray line in Figure 2a). In the numerical model, we calibrate parameters $L, F$, and $h$ using the least-square method to provide the nearest approximation of the experimental reflection spectrum. In the calculations, the following range of parameter variation was assumed, resulting from the accuracy of the SEM image: $L \in(810,830) \mathrm{nm}, F \in$ (0.46, 0.50), $H \in(175,205) \mathrm{nm}$. The calculated reflection spectrum (black line in Figure 2a), based on the calibrated parameters $L=820 \mathrm{~nm}, F=0.493$, and $h=198 \mathrm{~nm}$, shows very good agreement with the experimental measurements and, in particular, confirms very low power reflectance at the wavelength of $1050 \mathrm{~nm}$, mainly due to the deviation from the rectangular CS shape.

In the next step we redesigned the trapezoidal CS MHCG with a sidewall angle of $16^{\circ}$ as an invariant parameter to have the following optimal parameters: $L=820 \mathrm{~nm}, F=0.28, h=$ 
Table 1. Parameters of Designed and Realized MHCGs ${ }^{a}$

\begin{tabular}{|c|c|c|c|c|c|c|c|c|c|}
\hline \multirow[b]{2}{*}{ sample } & \multirow[b]{2}{*}{ material } & \multirow[b]{2}{*}{ nominal wavelength $(\mathrm{nm})$} & \multicolumn{3}{|c|}{ nominal parameter values } & \multicolumn{3}{|c|}{ parameters of fabricated samples } & \multirow[b]{2}{*}{ fabrication method } \\
\hline & & & $L(\mathrm{~nm})$ & $F$ & $h(\mathrm{~nm})$ & $L(\mathrm{~nm})$ & $F$ & $h(\mathrm{~nm})$ & \\
\hline A & $\mathrm{GaN}$ & 480 & 446 & 0.40 & 119 & 400 & 0.60 & 100 & EBL+ICP RIE \\
\hline B & $\mathrm{GaN}$ & 620 & 576 & 0.40 & 154 & 595 & 0.72 & 107 & EBL+ICP RIE \\
\hline $\mathrm{C}$ & $(\mathrm{Cd}, \mathrm{Zn}, \mathrm{Mg}) \mathrm{Te}$ & 880 & 791 & 0.38 & 184 & 760 & 0.68 & 184 & FIB \\
\hline $\mathrm{D}$ & $\mathrm{GaP}$ & 1550 & 1370 & 0.37 & 192 & 1350 & 0.95 & 500 & plasma FIB \\
\hline
\end{tabular}

${ }^{a}$ Nominal parameters of the MHCGs correspond to rectangular-shaped CS that were designed. Realized samples are not of rectangular CS (see Figure 2) and the fill factors $F$ of the samples correspond to the base widths of the stripes (see Figure $1 b$ ).
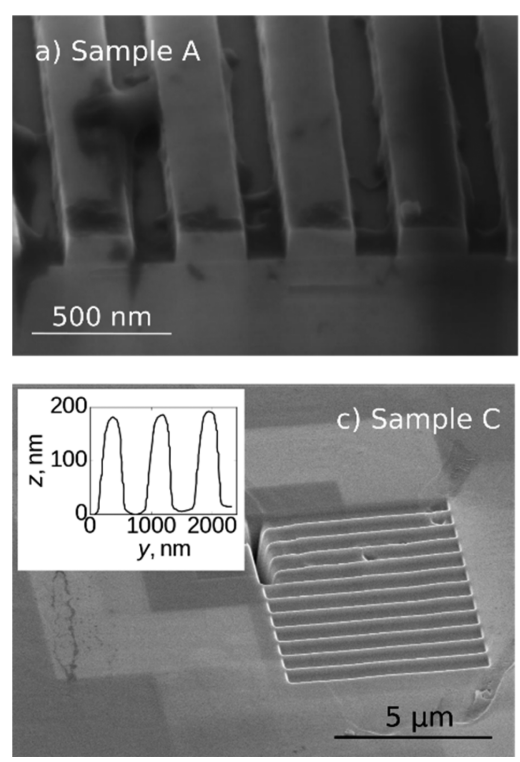
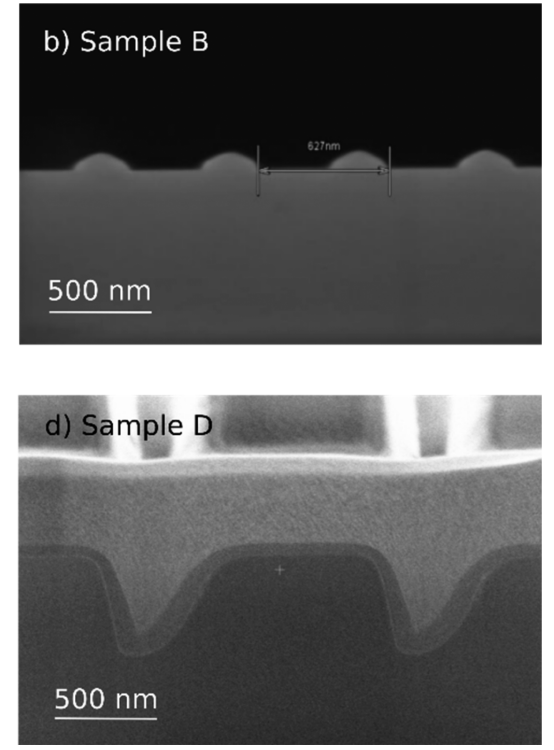

Figure 3. SEM images of realized MHCG mirrors for (a) sample A made of GaN, (b) sample B made of GaN, (c) sample C made of CdZnMgTe (inset, AFM surface scan profile), and (d) sample D made of GaP (coated with platinum).

$200 \mathrm{~nm}$. According to the numerical model, these parameters provide $97 \%$ reflection at $1050 \mathrm{~nm}$ (red line in Figure 2a). Figure $2 \mathrm{c}$ shows a SEM image of sample 2 fabricated according to the new design, with the following measured parameters: $L$ $=820 \pm 10 \mathrm{~nm}, F=0.26 \pm 0.02, h=190 \pm 11 \mathrm{~nm}$, and $\mathrm{a}$ sidewall angle of $14^{\circ}$. The measured reflectance was $95 \%$ (dark blue line in Figure 2a). The calculated reflection of sample 2 with the calibrated parameters $L=820 \mathrm{~nm}, F=0.265$, and $h=$ $197 \mathrm{~nm}$ is in close agreement with the experiment (dark red line in Figure 2a). As the previous example shows, the numerical analysis provides high accuracy for determining the reflected spectra of MHCGs. This example also shows the possibility for numerical adjustment of the MHCG parameters based on experimental CS, enabling nearly total power reflectance. It therefore raises the following question: Does any CS of an MHCG obtained experimentally allow for total reflection? What follows is an attempt to answer this question using our method of numerical analysis.

Four MHCGs made using different materials with rectangular CS were designed to provide maximal optical power reflectance at various operational wavelengths, corresponding to applications related to visible and infrared spectral ranges. Sample A was composed of $\mathrm{GaN}$ and designed for a wavelength of $480 \mathrm{~nm}$. Sample B was also composed of GaN and designed for a wavelength of $620 \mathrm{~nm}$. Samples A and B were processed using EBL and ICP-RIE. Sample C was composed of $(\mathrm{Cd}, \mathrm{Zn}, \mathrm{Mg}) \mathrm{Te}$ for a wavelength of $880 \mathrm{~nm}$ and fabricated with a gallium FIB system. Sample D was composed of $\mathrm{GaP}$, designed for a wavelength of $1550 \mathrm{~nm}$ and fabricated using a xenon plasma FIB system. The geometrical parameters of the designed (nominal parameters) and fabricated structures (parameters of the realized samples) are summarized in Table 1. The parameters of the fabricated MHCGs were estimated from the scanning electron microscope (SEM) images and atomic force microscope (AFM) scan profiles shown in Figure 3.

The period is a primary parameter responsible for the MHCG's wavelength of maximal optical power reflectance. The period of a grating can be controlled with the highest precision, regardless of the etching method used. The etching depth, however, is far more difficult to control precisely and has a significant influence on the MHCG power reflectance. A relative deviation in etching depth of $5 \%$ from the optimal value typically corresponds to a $1 \%$ drop in MHCG power reflectance at the designed wavelength. ${ }^{31}$ In ICP-RIE, the etching rate depends mostly on the temperature and pressure during the process, which determine the etching rate and anisotropy. ${ }^{23}$ A well-controlled etching depth can be obtained by properly tuning the etching conditions to slow the etch rate. This usually involves preconditioning the chamber and using reference samples to obtain a well-controlled and reproducible etch rate. Figure 3a,b shows samples A and B fabricated by ICP-RIE. They show well-defined stripes, between which the semiconductor surface is relatively flat. ICP-RIE therefore enables satisfactory control of the fill factor of the grating. The fill factor of the MHCG as well as the CS shape can be additionally tuned by ensuring proper exposure conditions 
during lithography, etching conditions, and the proper choice of mask material.

In FIB methods, the etching depth can be controlled more precisely and sidewall smoothness can be improved by optimizing numerous process parameters, the most important of which are accelerating voltage, beam current, dwell time, and charge removal. The parameters must be tuned for the particular material used in the sample. Increasing the precision of the etching depth and CS inevitably leads to longer process times, which may affect the precision of other MHCG parameters, especially in the case of larger area gratings. Figure 3c,d illustrates samples C and D, fabricated with FIB. In contrast to MHCGs fabricated with ICP-RIE, both the semiconductor surface between the stripes and the edges of the stripes are curved. This is related to the uneven distribution of energy from the ions hitting the semiconductor surface, as well as to the thermal drift of the samples during the milling process.

Figure 4 illustrates the CS of samples A-D implemented in the PWRTM software, reproduced based on the SEM and

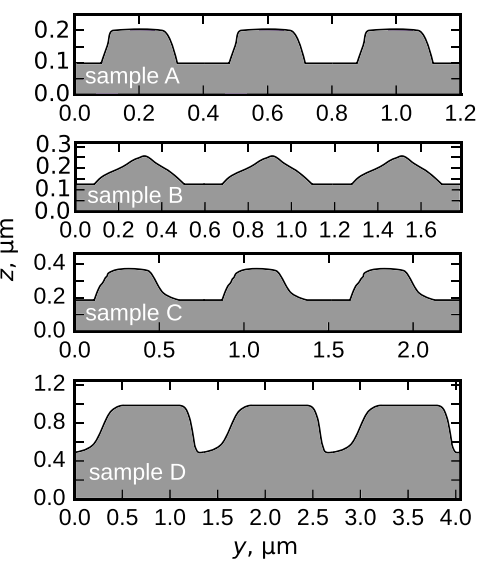

Figure 4. Cross-section profiles for samples A-D extracted from SEM or AFM images of the monolithic high contrast gratings shown in Figure 2, used as the inputs for calculations of the reflection spectra.

AFM images shown in Figure 3. The real-world profiles of the CS were used as the inputs for calculations of the reflection spectra illustrated in Figure 5. The reflection spectra calculated based on the extracted CS shapes and geometrical parameters of the realized samples (black lines) reveal power reflectance significantly below that expected for a CS designed to be perfectly rectangular (dark gray lines). This discrepancy is a result of the different shapes of the realized CS and the designed (nominal) CS. It is also due to their different geometrical parameters, which are collected for comparison in Table 1. Figure 6 presents power reflectance maps for each of the extracted MHCG profiles, illustrating power reflectance as a function of height and the fill factor of the gratings. The points labeled "realized" show the parameters of the fabricated samples. The points indicated "optimal" show parameters enabling maximal power reflectance close to $100 \%$ for realworld CS shapes and for the period of the realized samples (see Table 1). The maps demonstrate the possibility of tuning the MHCG parameters to improve power reflectance. However, multidimensional optimization was performed to obtain nearly $100 \%$ reflectivity for each CS of the fabricated MHCGs (see Figure 4), including optimization of the period, height, and the distance between the stripes. The parameters
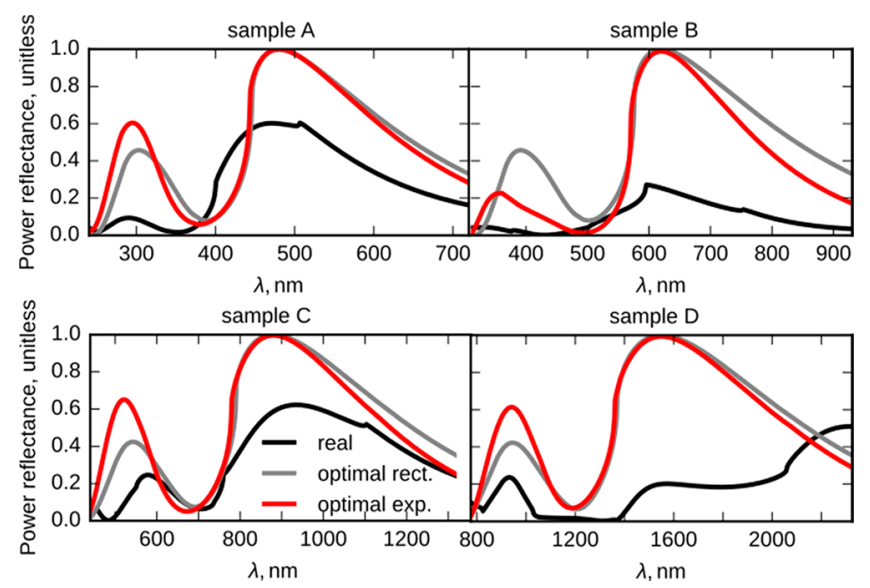

Figure 5. Calculated reflection spectra of monolithic high contrast gratings for designed rectangular cross sections (gray lines, see Table 1 for parameters), cross sections extracted from SEM and AFM images (black lines, see Table 1 for parameters), and cross sections extracted from SEM and AFM images, but with other parameters optimized to obtain maximized power reflectance (red lines, see Table 2 for parameters), for samples A-D shown in Figure 2. All reflection spectra are for normal illumination by TE polarized light.

for the optimization are summarized in Table 2. The red lines in Figure 5 show the power reflectance spectra of the samples with numerically optimized parameters for the experimentally achieved CS.

It is worth noting that, regardless of the shape of the CS or the material from which the mirror was made, the regions of high-power reflectance in the maps presented in Figure 6 as well as the optimal spectra shown in Figure 5 (gray and red lines) reveal similar shapes. A direct comparison of the spectra reveals differences of only a few nanometers in the spectral widths of the high reflectance stopband, at levels above $90 \%$ for the MHCGs with a rectangular-shaped CS compared to the nonrectangular shapes with optimal parameters. This suggests that the shape of the CS has a rather weak influence on the TE polarization reflection spectra. It is also worth noting that a fill factor ranging from approximately 0.4 to 0.5 is close to optimal, regardless of the CS shapes considered in the analysis. Therefore, modification of the fill factor in this range has a negligible effect on the maximal power reflectance. Figure 7 illustrates the optimal CS profiles of the samples and the corresponding light intensity distributions.

The following example, although not a strict proof, strengthens our conclusion that any CS shape of the periodically distributed stripes provides close to $100 \%$ reflection for either TE or TM polarization if the geometrical parameters of the stripes are properly adjusted. We optimize the geometric parameters of two arbitrarily designed asymmetrical shapes with rambling CS, as illustrated in Figure 8 (structures $\mathrm{K}$ and $\mathrm{T}$ ). The sidewalls of the stripes are not corrugated along the stripes, that is, in the $x$-direction. Corrugation along the stripes will be considered in Impact of Wall Corrugations on MHCG Power Reflectance.

In the calculations, we assume a refractive index of the MHCG equal to 3.5, corresponding to the refractive index of numerous narrow bandgap alloys in the infrared spectral range, including GaAs. For each of these contorted and nonsymmetrical shapes, we performed multidimensional optimization searching for maximal reflectance in TE and TM polarizations separately, taking the period, height, and fill factor as variable 

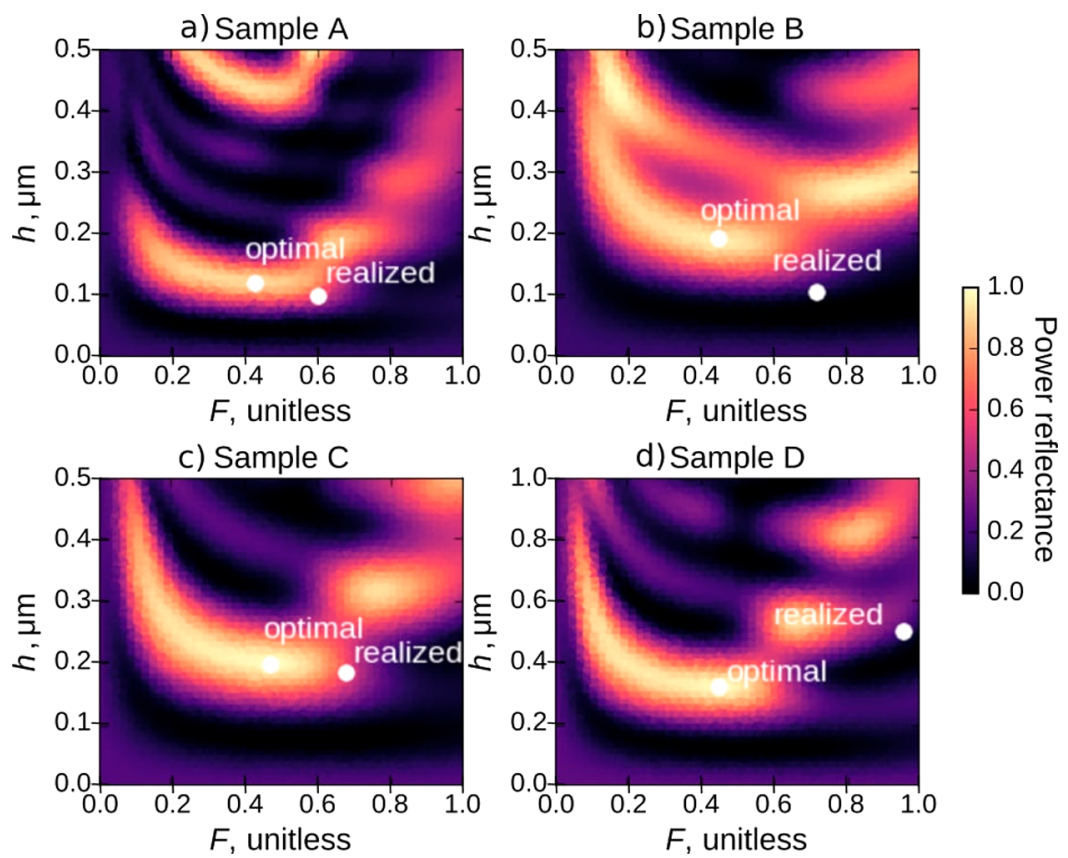

Figure 6. Calculated power reflectance maps for samples A-D under normal TE polarization. Power reflectance is shown as a function of the height of the stripes $(h)$ and the fill factor $(F)$ for a fixed value of the period of the grating. The values of the period were determined experimentally (see Table 1). For each sample, $h$ and $F$ indicated as "realized" are taken directly from the experimental results and $h$ and $F$ indicated as "optimal" are values ensuring the maximum power reflectance, while keeping the shape of the stripe and period determined in the experiment. See Figure S1 in the Supporting Information for the maps in the power reflectance range $>80 \%$.

Table 2. Parameters that Ensure Maximum Power Reflectance of the MHCG with Stripe Cross-Section Shapes, as Determined in the Experiment, but With Optimized $L, F$, and $h^{a}$

\begin{tabular}{cccccc} 
& & \multicolumn{4}{c}{ for TE polarization } \\
\cline { 3 - 6 } structure & wavelength $(\mathrm{nm})$ & $L(\mathrm{~nm})$ & $F$ & $h(\mathrm{~nm})$ & $R(\%)$ \\
A & 480 & 443 & 0.43 & 121 & 99.938 \\
B & 620 & 570 & 0.45 & 192 & 98.820 \\
C & 880 & 780 & 0.47 & 199 & 99.593 \\
D & 1550 & 1360 & 0.45 & 321 & 99.320
\end{tabular}

${ }^{a_{T}}$ The calculations were performed for a fixed wavelength and with TE polarized incident light.
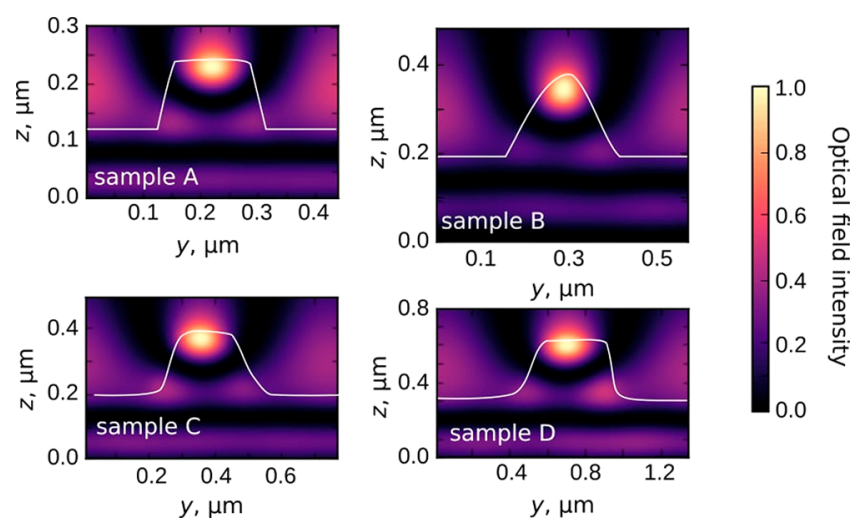

Figure 7. Optimal profiles of a single stripe for each of the analyzed monolithic high contrast gratings along with corresponding normalized optical field distributions (for the optimal parameters, see Table 2). In the simulations, TE polarized light was at normal incidence on the monolithic high contrast grating from the substrate. parameters. The fill factor is here defined as the maximal width of the stripe with respect to the period. As a result, we found the geometrical parameters of the structures (see Table 3) enabling $100 \%$ reflectivity for TE and TM polarizations. The power reflectance spectra calculated for these parameters are shown in Figure 9. The reflectance spectra are comparable in terms of the high reflectance stopband width. A slight difference can be noticed in the configurations designed for TE polarization. The spectrum produced by structure $\mathrm{K}$ is about $5 \mathrm{~nm}$ broader, at $90 \%$ reflectivity compared to structure $\mathrm{T}$. We attribute this difference to the greater roughness of the right sidewall in structure T. Surprisingly, there is no comparable difference in the case of TM polarization, where the field dominantly accumulates on the left and right walls of the stripes (Figures 8). Therefore, one would predict that the roughness of the walls should disturb the spectrum more strongly in the case of TM polarization than in the case of TE polarization. Although this analysis is not a strict proof, it shows that, regardless of how strongly the profile is deviated from a regular symmetric profile, if it is periodically repeated, the resulting MHCG can produce nearly $100 \%$ power reflectance. Therefore, the MHCG period and etching depth are the crucial parameters for maintaining nearly $100 \%$ power reflectance for a certain wavelength, whereas the CS profile is of less importance.

\section{OPTIMAL CROSS-SECTION DUE TO SPECTRUM WIDTH}

In the previous section, we showed that both of the CS shapes of the MHCG stripes that we investigated enable close to $100 \%$ reflectance. In this section, we search for the optimal CS shape of the stripes that enables the broadest possible reflectance spectrum. For this purpose, we consider various CS shapes, based on a trapezoid that with a top base that is 

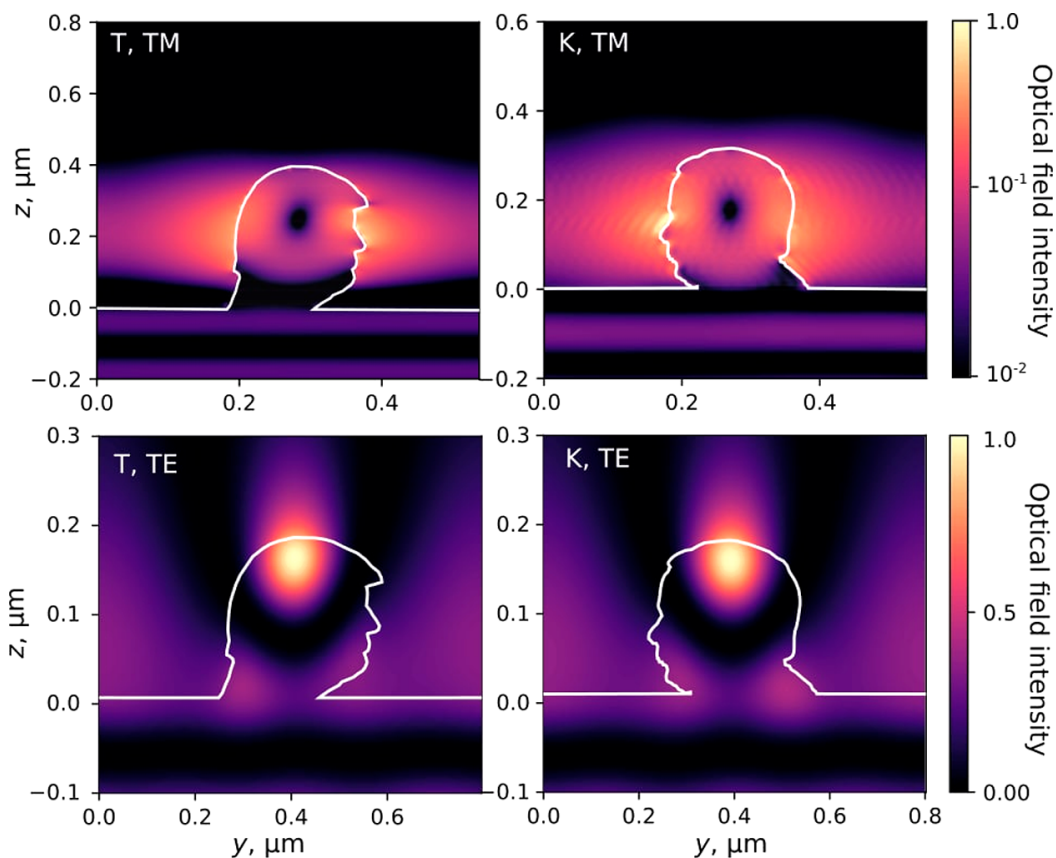

Figure 8. Optical field intensity distribution in a single stripe of a monolithic high contrast grating for structures $\mathrm{K}$ and $\mathrm{T}$. The structures are illuminated by normally incident TE and TM polarized light.

Table 3. Optimal Parameters of MHCGs for Grating Structures K and T with Quasi-Arbitrary Cross-Sections

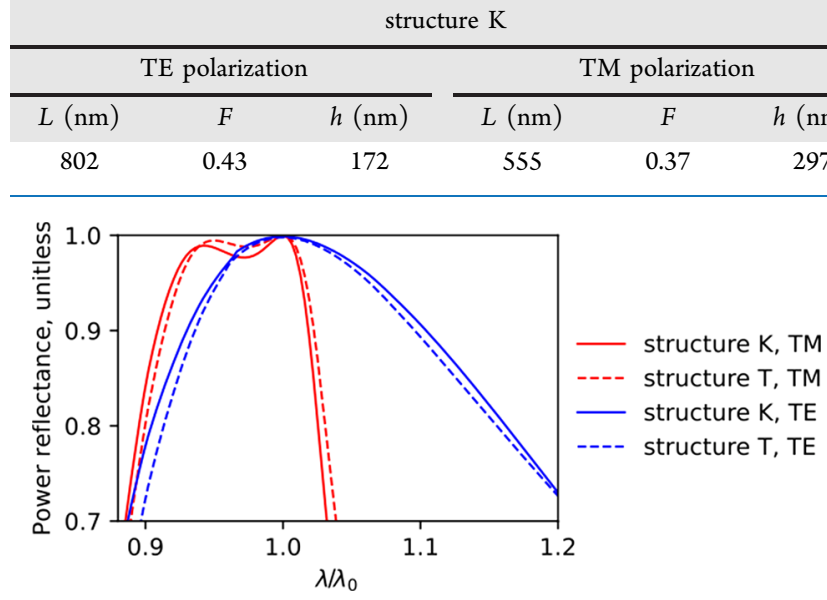

Figure 9. Optical power reflectance spectra of structures $\mathrm{T}$ and $\mathrm{K}$ (see Figure 7) in TE and TM configurations, illuminated by corresponding light polarizations; $\lambda_{0}$ is the wavelength of maximal power reflectance.

smaller than or equal to the bottom base and which is symmetrical with respect to the $y$-axis (Figure 10a). In our analysis, we use the parameter $k$, which is the ratio of the lengths of top and the bottom bases of the trapezoid (see Figure 10). Thus, $k=1$ corresponds to a rectangle and $k=0$ corresponds to a triangle. We also consider modifications of the trapezoidal shape with convex (Figure 10b) and concave legs (Figure 10c), where parameter $k$ also ranges from 0 to 1 . The curvature of the walls is expressed by simple mathematical formulas describing the right walls of the stripes if the center of the bottom base is the point $(y, z)=(0,0)$ :

$$
z(y)=h\left(1-\left(\frac{2 y-k a}{a-k a}\right)^{2}\right)
$$

in the case of convex sidewalls and

\begin{tabular}{|c|c|c|c|c|c|}
\hline \multicolumn{3}{|c|}{ TE polarization } & \multicolumn{3}{|c|}{ TM polarization } \\
\hline$L(\mathrm{~nm})$ & $F$ & $h(\mathrm{~nm})$ & $L(\mathrm{~nm})$ & $F$ & $h(\mathrm{~nm})$ \\
\hline 796 & 0.42 & 175 & 537 & 0.37 & 382 \\
\hline
\end{tabular}
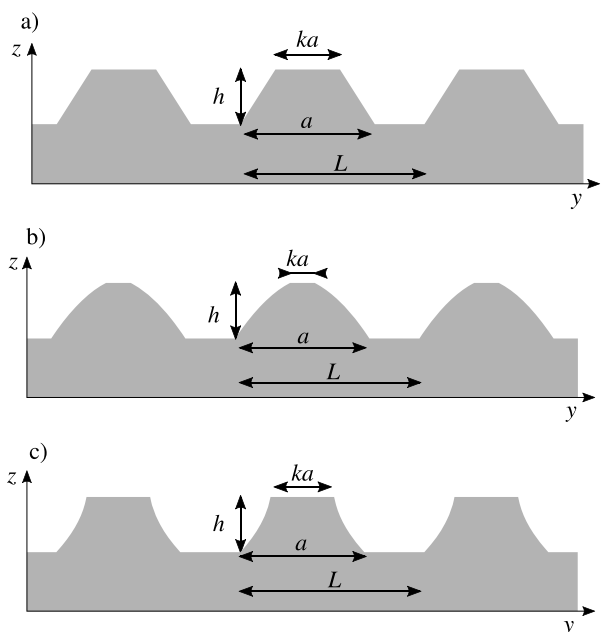

Figure 10. MHCG structures with trapezoid CS shapes with (a) straight, (b) convex, and (c) concave sidewalls. The parameter $k$ is defined as the ratio of the length of the upper base to the length of the lower base.

$$
z(y)=h\left(1-\sqrt{\frac{2 y-k a}{a-k a}}\right)
$$

in the case of concave sidewalls. For $k=1$, the sidewalls are vertical. We do not consider structures where $k>1$, which would correspond to stripes that have an undercut. 


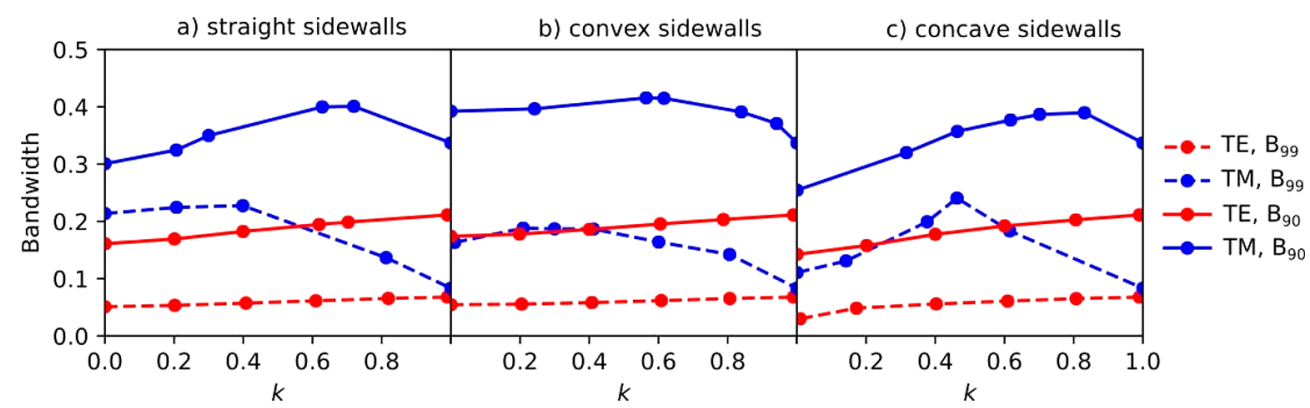

Figure 11. Dependence of the reflection stopbands $B_{90}$ (solid lines) and $B_{99}$ (dashed lines) on parameter $k$ for TE (red lines) and TM (blue lines) polarizations and for different cross-sections in (a)-(c) illustrated in Figure 9.

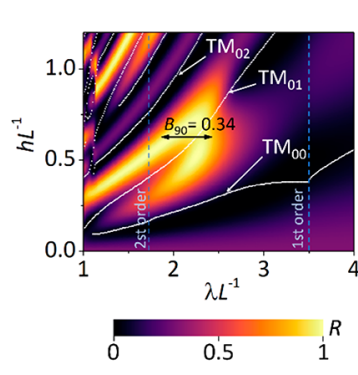

a)

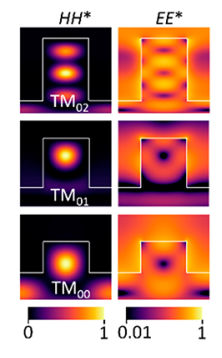

b)

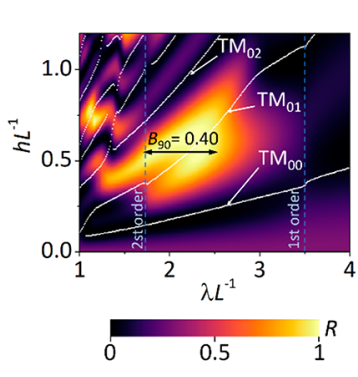

c)

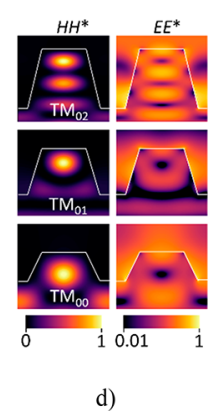

Figure 12. Power reflectance maps of TM polarization for $k=1, F=0.55$ (a) and $k=0.6, F=0.8$ (c) with dispersion curves of leaky TM modes indicated by white lines in (a) and (c). The square of the magnetic $\left(H H^{*}\right)$ and electric $\left(E E^{*}\right)$ components of the electromagnetic field are plotted in (b) and (d). The $H H^{*}$ is represented in linear, while $E E^{*}$ is in the logarithmic scale of colors. Vertical dashed lines in (a) and (c) identify boarders of successive diffraction orders emerging in the substrate.

Trapezoidal CS shapes can be a consequence of the lithographic and etching process. Sloped sidewalls are observed when isotropic etching (wet etching or RIE) is used. In wet etching, the slope of the sidewalls depends on the etchant and the crystallographic orientation of the sample. ${ }^{18}$ In dry etching, the slope depends on the conditions of the etching process, in particular, the temperature and the content of chemical radicals. The higher the temperature and the higher the concentration of chemical radicals, the less anisotropic the etching and the steeper the slope of the sidewalls. ${ }^{23}$ Sloping sidewalls can also be intentionally fabricated using gray scale lithography. ${ }^{32,33}$ In the FIB milling process, the slope of the sidewalls depends on the power density distribution in the ion beam and the processed material.

We performed optimization of the CS shapes presented in Figure 10 with respect to maximal bandwidths $(B)$ of MHCG power reflectance spectra of $90 \%\left(B_{90}\right)$ and $99 \%\left(B_{99}\right)$, separately. The choice of two levels of optical power reflectance relates to two important applications of micromirrors. The first level of $90 \%$ relates to mirrors used in microelectromechanical (MEMS) applications, ${ }^{34}$ whereas the second level of above $99 \%$ relates to mirrors used in VCSELs. ${ }^{24}$ The bandwidth is defined as $B=0.5\left(\lambda_{\mathrm{H}}-\lambda_{\mathrm{L}}\right) /$ $\left(\lambda_{\mathrm{H}}+\lambda_{\mathrm{L}}\right)$, where $\lambda_{\mathrm{H}}$ and $\lambda_{\mathrm{L}}$ are the higher and lower wavelength limits, respectively, between which the power reflectance is more than $90 \%\left(B_{90}\right)$ and more than $99 \%\left(B_{99}\right)$. In our optimization procedure, for each configuration shown in Figure 10, we calculate power reflectance maps for the domain of the wavelength $\lambda$ in a range from 0 to $3 L$ with a step of $0.06 L$, for stripe heights $h$ in the range from 0 to $2 L$ with a $0.04 L$ step, for $k$ in the range from 0 to 1 with a 0.1 step, and for $F$ in the range from 0.1 to 0.9 with a 0.1 step (see Supporting Information, Figures S2-S7, for all calculated maps). The observed local maxima are used as starting points in a multidimensional optimization procedure, which takes $L$, $F, h$, and $k$ as variable parameters. Figure 11 presents the dependence between the bandwidths $\left(B_{90}\right.$ and $\left.B_{99}\right)$ and the $k$ parameter for gratings with straight, concave, and convex sidewalls for both TE and TM polarized light. The values providing the widest spectra are collected in Tables $\mathrm{S} 1-\mathrm{S} 3$ in the Supporting Information.

For TE polarization, $B_{90}$ and $B_{99}$ are slowly increasing functions with increasing parameter $k$. When $k=1.0$, both $B_{90}$ and $B_{99}$ are maximum, revealing that a rectangular CS is optimal for reflection of TE polarized light. The corresponding reflection spectra for maximal stopbands of $90 \%$ and $99 \%$ are plotted in Figure S8 in the Supporting Information. TM polarization enables significantly broader reflectance spectra than TE polarization. For TM polarization, $B_{90}$ is above 0.3, regardless of $k$. The maximal values of $B_{90}$ are obtained for a relatively broad range of $k$ from 0.6 to 0.8 . In this range, $B_{90}$ reaches the maximal level of 0.4 . $B_{99}$ reaches its maximum above 0.2 for $k$ close to 0.3 . Similar dependence is observed for configurations with concave and convex sidewalls.

Figures S9 and S10 in the Supporting Information combine reflectivity maps for TM and TE polarizations, respectively, for CS shapes with straight sidewalls (see Figure 10a). Similar reflectivity maps can be plotted for CS with concave and convex sidewalls. The maps illustrate reflectivity as a function of the normalized height of the stripes (with respect to the grating period) and the inverted wavelength for different values of $k$. The maps are shown for the fill factor $F$ which is close to the value of $F$ for the largest possible $B_{90}(F=0.8$ for TM polarization and $F=0.3$ for TE polarization). The size and shape of the high reflectivity region is dependent on the dispersion curves of leaky modes present in the structure. 
Figure 12 illustrates two examples of TM reflectance maps for optimal MHCGs with rectangular (Figure 12a) and trapezoidal (Figure 12c) CS, together with the dispersion curves of the modes. The numerical identification of the dispersion curves is significantly hindered by the very low $Q$-factor of the leaky modes, which become untraceable for eigenvalue solvers. ${ }^{35}$ Therefore, we identify the modes by the buildup of the light intensity inside the MHCG as minima of the formula:

$$
D=\frac{\partial^{2}}{\partial h^{2}} \mathfrak{I}=\frac{\partial^{2}}{\partial h^{2}} \int_{\text {MHCG }} H H^{*} \mathrm{~d} y \mathrm{~d} z
$$

where $H$ is the magnetic component of the electromagnetic field that is integrated over the grating layer.

The presence of leaky modes in the structure impedes their straightforward identification by a maxima of $\mathfrak{J}$ and therefore their localization by second derivative minima of $\mathfrak{I}$ is significantly more effective. The choice of $H$ facilitates efficient integration, since $H$ is a slowly varying function inside the MHCG. In contrast, the electric field ( $E$; see Figure $12 \mathrm{~b}, \mathrm{~d}$ ) reveals large gradients at the sidewalls of the grating. The proposed method of identifying the modes is in exact agreement with theory ${ }^{36}$ in the benchmark case of a membrane grating (HCG). Figure 12a,c shows that the largest region of very high reflection is constrained by the $\mathrm{TM}_{00}$ and $\mathrm{TM}_{02}$ modes and is crossed by the $\mathrm{TM}_{01}$ mode. The size and shape of the high reflectivity region is thus dependent on the position of the dispersion curves of $\mathrm{TM}_{00}$ and $\mathrm{TM}_{02}$, which is in close analogy with the formation of the highly reflective region in the case of an HCG. ${ }^{37}$ The broad reflection spectrum is strongly dependent on $F$. The largest area of strong reflection is obtained when the ratio between the volume of stripes and air is close to $50 \%$ in the grating layer, as shown in Figure S2 in the Supporting Information, and decreases when $F$ increases. The area of strong reflection is extended in the direction determined by the dispersion curves of the modes between which it is located. In order for this area to be more parallel to the $\lambda L^{-1}$ axis, the dispersion curves of $\mathrm{TM}_{00}$ and $\mathrm{TM}_{02}$ must also be parallel to this axis. This can be achieved by increasing the effective refractive index of the modes, ${ }^{36}$ which requires increasing $F$, thereby reducing the size of the highly reflective region and also reducing the maximal reflection. The trapezoidal CS contributes to slightly more accumulation of $\mathrm{TM}_{00}$ in the stripe in comparison to the rectangular CS. This effect manifests itself in a dispersion curve of the $\mathrm{TM}_{00}$ that is somewhat more parallel to the horizontal axis. As a result, the area of high reflection is aligned more in parallel to the $\lambda L^{-1}$ axis, providing a wide reflection spectrum. The spectrum is broader than that of the rectangular CS, despite the pronounced effect of light leakage to the second diffraction order. This noticeably lowers the reflection into the zeroth order. The leakage to the second order is $10 \times$ weaker in the rectangular CS and, hence, invisible in Figure 12a.

Figure 13 illustrates the reflectance spectra of three trapezoidal MHCGs with a refractive index of 3.5. The two configurations of the MHCG were optimized with respect to their maximal reflection stopbands, of 0.99 (MHCG1, red line) and 0.999 (MHCG2, orange line). The third configuration was optimized with respect to the maximal reflection spectrum integral above the reflection level of 0.99 (MHCG3, yellow line). The spatial parameters of these gratings are listed in Table 4. MHCG1 and MHCG3 show reflection spectra with pronounced minima at the reflection level of 0.99 and 0.997 ,

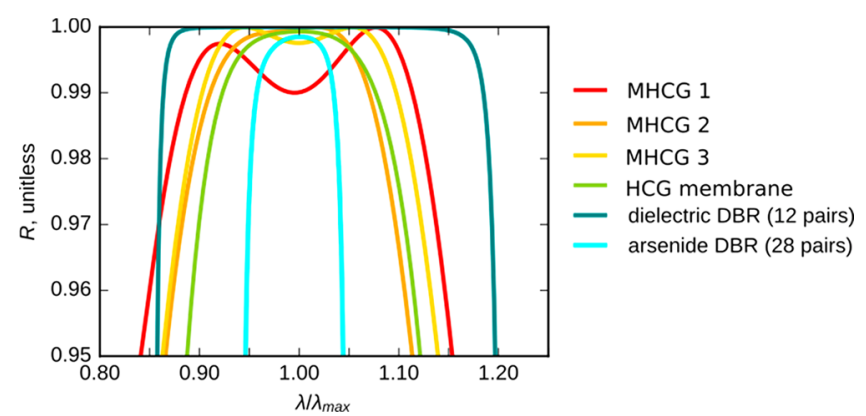

Figure 13. Simulated power reflectance spectrum for MHCG configurations with trapezoidal CS for TM polarization, providing the broadest spectra for: reflectance above 99\% (red line, MHCG1); reflectance above $99.9 \%$ (orange line, MHCG2); the largest integral of reflection spectrum above 99\% (yellow line, MHCG3). The figure also illustrates the simulated reflectance spectra for: an HCG membrane with TM polarization (light green line); a dielectric $\mathrm{SiO}_{2} / \mathrm{TiO}_{2}$ DBR (dark green line); a semiconductor GaAs/ $\mathrm{Al}_{0.9} \mathrm{Ga}_{0.1} \mathrm{As}$ DBR (light blue line).

Table 4. Optimal Parameters $(k, L, F$, and $h)$ and Value of the Reflection Bandwidth at a Power Reflectance Level of 99\% $\left(B_{99}\right)$ for MHCGs with a Trapezoidal Cross-Section of Straight Sidewalls Optimized under Different Assumptions, Listed in the Second Column ${ }^{a}$

$\begin{array}{ccccccc} & \begin{array}{c}\text { optimization } \\ \text { condition }\end{array} & k & L & F & H & B_{99} \\ \text { MHCG1 } & \begin{array}{c}\text { maximal reflection } \\ \text { stopband for } \\ R=0.99\end{array} & 0.4 & 0.44 \lambda & 0.87 & 0.26 \lambda & 0.228 \\ \text { MHCG2 } & \begin{array}{c}\text { maximal reflection } \\ \text { stopband for } \\ R=0.999\end{array} & 0.403 & 0.45 \lambda & 0.87 & 0.29 \lambda & 0.159 \\ \text { MHCG3 } & \begin{array}{c}\text { maximal reflection } \\ \text { spectra integral } \\ \text { above } R=0.99\end{array} & 0.22 & 0.44 \lambda & 1.0 & 0.37 \lambda & 0.196 \\ & & & & & \end{array}$

${ }^{a}$ All gratings were optimized for normally incident TM polarized light and the refractive index of the grating $n=3.5$.

respectively. The reflection spectrum of MHCG2 does not show a local minimum, and closely resembles the spectral shape for a DBR. For comparison, the optical power reflectance spectra of an HCG membrane (light green line), a dielectric DBR (dark green line), and a semiconductor DBR (blue line) are also shown in the figure. The refractive index of the HCG membrane is also 3.5 and the membrane is suspended in air. The CS of the HCG membrane is rectangular and the optimal parameters with respect to the maximal reflection stopband for $R=0.99$ were found using the procedure applied for optimization of the MHCG described in Optimal Cross-Section Due to Spectrum Width. The semiconductor DBR is composed of 28 pairs of quarter-wavelengththick layers, with refractive indices of 3.5 and 3.0 corresponding to the refractive indices of $\mathrm{GaAs}$ and $\mathrm{Al}_{0.9} \mathrm{Ga}_{0.1} \mathrm{As}$ at the wavelength of $980 \mathrm{~nm}$. The dielectric DBR is composed of 12 pairs of quarter-wavelength-thick layers with refractive indices of 1.5 and 2.4, corresponding to the refractive indices of $\mathrm{SiO}_{2}$ and $\mathrm{TiO}_{2}$ at the same wavelength, which enables one of the largest refractive index contrasts between the DBR layers.

Surprisingly, the reflection spectra of the three trapezoidal MHCGs were significantly broader (see Table 4 for values of $\left.B_{99}\right)$ than those of both the membrane HCG $\left(B_{99}=0.152\right)$, which was close to the spectrum reported in ref 26 and the 

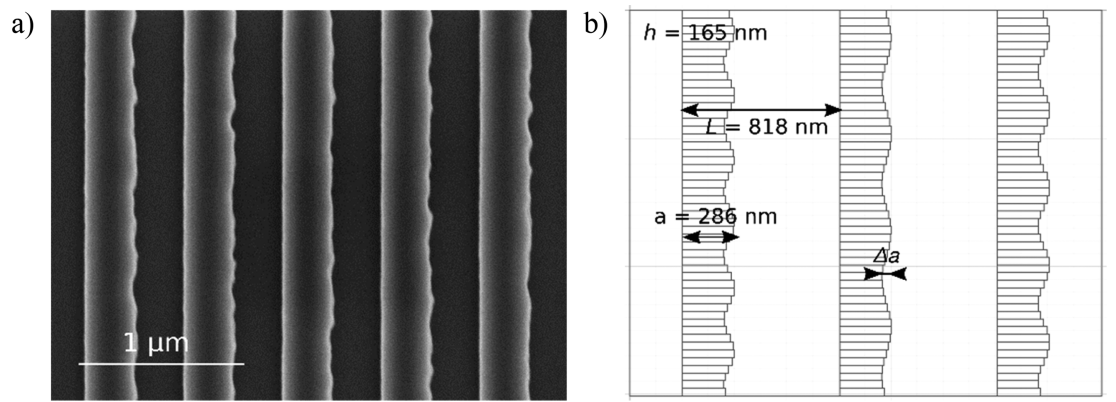

Figure 14. (a) SEM image of the GaAs-based MHCG structure showing corrugation of the side edges of the MHCG strips; (b) top view of the MHCG structure for which the 3D calculations were performed. Periodic boundary conditions are imposed from all sides in the grating plane. All three stripes have the same period, height, and fill factor. They differ in the period and amplitude $(\Delta a)$ of the corrugation, which is described by a sine function.

semiconductor DBR $\left(B_{99}=0.075\right)$. However, the dielectric DBR exhibited the broadest spectrum $\left(B_{99}=0.311\right)$ of all the considered mirror configurations. This example demonstrates that MHCGs with intentionally tuned CS shapes can outperform HCGs and semiconductor DBRs in terms of their reflection properties. The widespread belief that MHCGs exhibit narrower reflection spectra than HCGs and semiconductor DBRs is thus no longer justified.

\section{IMPACT OF WALL CORRUGATIONS ON MHCG POWER REFLECTANCE}

The fabrication inaccuracy of MHCGs may result not only in stripes with nonperfect CS deviating from the assumed design, but also in corrugation of the sidewalls along the stripes (along the $x$-axis, see Figure 1). In this section, we present an analysis of longitudinal sidewall corrugation, using the example of an MHCG fabricated from GaAs and designed for a wavelength of $980 \mathrm{~nm}$ with TE polarization. The considered MHCG was fabricated using EBL followed by ICP-RIE etching. A detailed description of the process can be found in ref 7. Figure 14a presents a top view SEM image of the MHCG, showing longitudinal corrugation of the side edges of the stripes. In this example, side corrugation is a consequence of imprecise control of the electron beam during the EBL exposure process. However, similar corrugations are inherent in all other fabrication methods and can affect both sidewalls.

To study the impact of longitudinal corrugation of the sidewalls on the reflectivity of the MHCG, we reproduced the corrugated structure in our numerical model, as illustrated in Figure $14 \mathrm{~b}$. The calculations were performed for three MHCG stripes with periodic boundary conditions and the following MHCG parameters: grating period $L=818 \mathrm{~nm}$, stripe height $h$ $=165 \mathrm{~nm}$, and averaged fill factor $F=0.35$ for TE polarization. In the simulation, a single period with corrugated walls and periodic boundary conditions was capable of providing $100 \%$ reflectance provided the parameters of the grating were chosen properly. This extends the conclusions presented in Influence of Cross-Sectional Shape on the Maximal Power Reflectance of the MHCG, showing that corrugations along the $x$-axis, if they recur periodically in subsequent stripes, do not deteriorate the reflectivity. At least three stripes of various corrugation are necessary to accurately reproduce power reflectance deterioration by a stochastic distribution of corrugation along the stripes. The CS of the stripes was rectangulars, representing the real shape presented in. ${ }^{9}$ The corrugation of the sidewalls was modeled using a sine function with a different period in each stripe, to represent the nonperiodic occurrence of the corrugation. We estimated the size of the ripples along the grating stripe edge as $\Delta a=50 \mathrm{~nm}$. This corresponds to the largest deviation visible in the SEM image. The calculations were performed in a three-dimensional modeling domain.

Figure 15 shows the reflection spectrum for the structure presented in Figure 14b (green line). This spectrum is

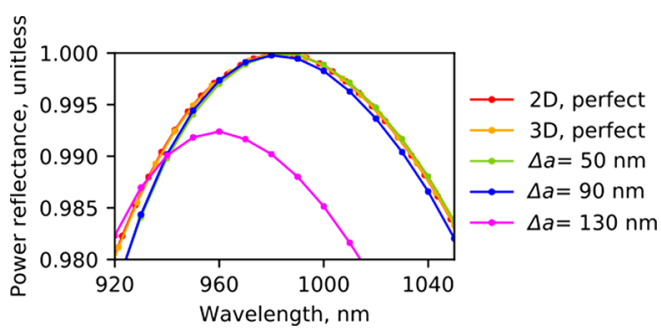

Figure 15. Power reflectance spectra calculated for a structure consisting of three stripes enclosed by periodic boundary conditions, each with a different level of corrugation (green, blue, and magenta lines) defined by $\Delta a$, and for a single stripe without corrugation enclosed by periodic boundary conditions calculated by twodimensional (2D, red line) and three-dimensional (3D, orange line) models.

compared with the spectra calculated for a single smooth stripe with periodic boundary conditions in $2 \mathrm{D}$ and $3 \mathrm{D}$ models (red and orange lines), revealing close results. A corrugation with $50 \mathrm{~nm}$ amplitude has no visible effect on the spectral width or the maximum reflectivity. A slight narrowing of the reflection spectrum (in the order of a few nanometers for a reflectivity of 0.98 ) is observed for a corrugation with $90 \mathrm{~nm}$ amplitude. The effect of such imperfections on reflectivity may be experimentally imperceptible. For a disturbance in the stripe sidewall shape to have an impact on reflectivity, its amplitude should be significantly more than $100 \mathrm{~nm}$ in this type of grating, corresponding to a wavelength of nearly $1 \mu \mathrm{m}$. With a corrugation amplitude of $130 \mathrm{~nm}$ (which corresponds to $40 \%$ of the stripe width and to $16 \%$ of the MHCG period), we observe a reduction in reflectivity from 1 to 0.99 and narrowing of the reflection spectrum by $50 \mathrm{~nm}$ for an optical power reflectance of 0.98 . Such severe corrugations were not observed in the gratings fabricated using standard processing methods discussed in this paper. It can be concluded that typical deviations in the sidewalls that occur during the processing of subwavelength gratings do not significantly affect the reflectivity of MHCGs. However, it is important to note that the analysis shown in Optimal Cross-Section Due to 
Spectrum Width and Impact of Wall Corrugations on MHCG Power Reflectance is only valid if the real-world MHCG with an arbitrary CS is periodic. Nonidentical CS of stripes or deviations in the transversal dimensions between the stripes of an MHCG at more than $15 \%$ of the period significantly deteriorate the optical power reflectivity of the MHCG.

\section{CONCLUSIONS}

In this work, we have shown that MHCG stripes do not need to have perfectly rectangular or even symmetric cross-sections to provide optical power reflectance close to $100 \%$. This greatly relaxes the requirements on the fabrication of MHCG mirrors, which can be made from almost any semiconductor material used in optoelectronics. We have also demonstrated that MHCG mirrors with trapezoidal cross-sectional shapes can be obtained with a very wide reflectance spectrum of $99 \%$ using standard etching methods, provided that the etching parameters are carefully tuned. The width of the reflection spectrum of the MHCG is $20 \%$ of the operational wavelength, which is wider than the width of arsenide-based DBRs (7.5\%) and HCGs (15\%). At the same time, the MHCG is much thinner than a dielectric DBR mirror and less susceptible to mechanical damage than HCG membranes. Finally, we have shown that sidewall corrugations in the MHCG stripes have only a moderate impact on MHCG reflectance, if the amplitude of the corrugation of the sidewalls is less than $16 \%$ of the MHCG period. Sidewall corrugations of this amplitude or smaller occur due to fabrication inaccuracy during two-step processes, such as inductively coupled plasma reactive ion etching preceded by electron beam lithography and one-step processes such as focus ion beam lithography. However, we found that this level of corrugation does not affect either the maximal power reflectance or the reflectance spectrum of MHCGs significantly.

At a more general level, this work opens a new perspective for photonics development, in which totally reflecting mirrors of subwavelength thickness can be fabricated from a multitude of nonabsorbing materials using currently existing surface shaping technologies. This facilitates monolithic integration, enabling polarization selectivity and versatile phase tuning. Our main finding is that the quality of MHCG mirrors depends more on their periodicity than on the shape of individual grating stripes. However, if the cross-sections of the stripes are designed carefully MHCGs can outperform current semiconductor-based mirrors.

\section{ASSOCIATED CONTENT}

\section{SI Supporting Information}

The Supporting Information is available free of charge at https://pubs.acs.org/doi/10.1021/acsphotonics.1c00850.

Reflectivity maps for experimental structures A-D corresponding to Figure 5 for power reflectance above $80 \%$; Reflectivity maps as a function of wavelength and stripe height for structures with straight, concave, and convex sidewalls for different values of parameter $k$ and fill factors; Table with spatial parameters of optimal configurations corresponding to Figure 11; Power reflectance spectra of selected configurations; Reflectivity maps as a function of wavelength and stripe height for selected structures with straight sidewalls, illustrating modification of reflectance induced by changes in the $k$ parameter (PDF)

\section{AUTHOR INFORMATION}

\section{Corresponding Author}

Tomasz Czyszanowski - Photonics Group, Institute of Physics, Lodz University of Technology, 90-924 Łódź, Poland; 10 orcid.org/0000-0002-0283-5074;

Email: tomasz.czyszanowski@p.lodz.pl

\section{Authors}

Magdalena Marciniak - Photonics Group, Institute of Physics, Lodz University of Technology, 90-924 Łódź, Poland

Tsung-Scheng Chang - Department of Photonics, College of Electrical and Computer Engineering, National Yang Ming Chiao Tung University, Hsinchu 30010, Taiwan; (1) orcid.org/0000-0003-1418-312X

Tien-Chang Lu - Department of Photonics, College of Electrical and Computer Engineering, National Yang Ming Chiao Tung University, Hsinchu 30010, Taiwan; (1) orcid.org/0000-0003-4192-9919

Filip Hjort - Photonics Laboratory, Department of Microtechnology and Nanoscience, Chalmers University of Technology, SE-41296 Göteborg, Sweden; 이이이. 0000-0003-3694-3644

Åsa Haglund - Photonics Laboratory, Department of Microtechnology and Nanoscience, Chalmers University of Technology, SE-41296 Göteborg, Sweden

Eucja Marona - Institute of High Pressure Physics PAS, 01142 Warsaw, Poland; $\odot$ orcid.org/0000-0002-7432-5735

Matusz Gramala - Nanores Sp. z o.o. Sp. k., 53-317 Wroctaw, Poland; Department of Semiconductor Materials Engineering, Wroctaw University of Science and Technology, 50-370 Wroctaw, Poland

Paweł Modrzyński - Nanores Sp. z o.o. Sp. k., 53-317 Wroctaw, Poland; Department of Semiconductor Materials Engineering, Wroctaw University of Science and Technology, 50-370 Wroctaw, Poland

Robert Kudrawiec - Department of Semiconductor Materials Engineering, Wrocław University of Science and Technology, 50-370 Wrocław, Poland; 이이. org/0000-0003-25939172

Krzysztof Sawicki - Institute of Experimental Physics, Faculty of Physics, University of Warsaw, 02-093 Warsaw, Poland; (1) orcid.org/0000-0003-1617-2678

Rafał Bozek - Institute of Experimental Physics, Faculty of Physics, University of Warsaw, 02-093 Warsaw, Poland

Wojciech Pacuski - Institute of Experimental Physics, Faculty of Physics, University of Warsaw, 02-093 Warsaw, Poland; (1) orcid.org/0000-0001-8329-5278

Jan Suffczyński - Institute of Experimental Physics, Faculty of Physics, University of Warsaw, 02-093 Warsaw, Poland; (1) orcid.org/0000-0003-3737-464X

Marcin Gębski - Photonics Group, Institute of Physics, Lodz University of Technology, 90-924 Łódż, Poland

Artur Broda - Łukasiewicz Research Network - Institute of Microelectronics and Photonics, 02-668 Warsaw, Poland

Jan Muszalski - Łukasiewicz Research Network - Institute of Microelectronics and Photonics, 02-668 Warsaw, Poland

James A. Lott - Technical University Berlin, Institute of SolidState Physics, 10623 Berlin, Germany

Complete contact information is available at:

https://pubs.acs.org/10.1021/acsphotonics.1c00850

\section{Notes}

The authors declare no competing financial interest. 


\section{ACKNOWLEDGMENTS}

This work is supported by the Polish National Science Centre within the projects Opus (2018/29/B/ST7/01927, 2020/39/ B/ST7/03502, 2014/15/B/ST7/05258) and Preludium (2018/29/N/ST7/02460). T.-C.C. and T.-C.L. would like to acknowledge the support from the Ministry of Science and Technology in TAIWAN under Contract No. MOST 1092221-E-009-150, and MOST 109-2124-M-009-005. F.H. and Å.H. received financial support from the Swedish Research Council (2018-00295, 2017-04440) and their work was done in part at Myfab Chalmers.

\section{REFERENCES}

(1) Mateus, C. F. R.; Huang, M. Y. C.; Deng, Y.; Neureuther, A. R.; Chang-Hasnain, C. J. Ultrabroadband mirror using low-index cladded subwavelength grating. IEEE Photonics Technol. Lett. 2004, 16 (2), $518-520$.

(2) Boutami, S.; Benbakir, B.; Leclercq, J. L.; Viktorovitch, P. Compact and polarization controlled $1.55 \mu \mathrm{m}$ vertical-cavity surfaceemitting laser using single-layer photonic crystal mirror. Appl. Phys. Lett. 2007, 91 (7), 071105.

(3) Gȩbski, M.; Dems, M.; Szerling, A.; Motyka, M.; Marona, Ł.; Kruszka, R.; Urbańczyk, D.; Walczakowski, M.; Pałka, N.; WójcikJedlińska, A.; Wang, Q. J.; Zhang, D. H.; Bugajski, M.; Wasiak, M.; Czyszanowski, T. Monolithic high-index contrast grating: a material independent high-reflectance VCSEL mirror. Opt. Express 2015, 23 (9), 11674-11686.

(4) Marciniak, M.; Gębski, M.; Dems, M.; Haglund, E.; Larsson, A.; Riaziat, M.; Lott, J. A.; Czyszanowski, T. Optimal parameters of monolithic high contrast grating mirrors. Opt. Lett. 2016, 41 (15), 3495-3498.

(5) Komar, P.; Gębski, M.; Czyszanowski, T.; Dems, M.; Wasiak, M. Planar focusing reflectors based on monolithic high contrast gratings: design procedure and comparison with parabolic mirrors. Opt. Express 2020, 28 (26), 38745-38761.

(6) Kim, S.; Wang, Z.; Brodbeck, S.; Schneider, C.; Höfling, S.; Deng, H. Monolithic High-Contrast Grating Based Polariton Laser. ACS Photonics 2019, 6 (1), 18-22.

(7) Gębski, M.; Lott, J. A.; Czyszanowski, T. Electrically injected VCSEL with a composite DBR and MHCG reflector. Opt. Express 2019, 27 (5), 7139-7146.

(8) Marciniak, M.; Broda, A.; Gȩbski, M.; Dems, M.; Muszalski, J.; Czerwiński, A.; Ratajczak, A.; Marona, Ł.; Nakwaski, W.; Lott, J. A.; Czyszanowski, T. Tuning of reflection spectrum of a monolithic highcontrast grating by variation of its spatial dimensions. Opt. Express 2020, 28 (14), 20967-20977.

(9) Czyszanowski, T.; Gȩbski, M.; Pruszyńska-Karbownik, E.; Wasiak, M.; Lott, J. A. Monolithic high-contrast grating planar microcavities. Nanophotonics 2020, 9 (4), 913-925.

(10) Kishino, K.; Unlu, M. S.; Chyi, J. I.; Reed, J.; Arsenault, L.; Morkoç, H. Resonant cavity enhanced photodetectors. IEEE J. Quantum Electron. 1991, 27, 2025-34.

(11) Bravo-Abad, J.; Rodriguez, A.; Bermel, P.; Johnson, S. J.; Joannopoulos, J. D.; Soljačić, M. Enhanced nonlinear optics in photonic-crystal microcavities. Opt. Express 2007, 15, 16161-76.

(12) Schneider, C. A.; Heindel, T.; Huggenberger, A.; Niederstrasser, T. A.; Reitzenstein, S.; Forchel, A.; Höfling, S.; Kamp, M. Microcavity enhanced single photon emission from an electrically driven site-controlled quantum dot. Appl. Phys. Lett. 2012, 100, 091108.

(13) Ściesiek, M.; Sawicki, K.; Pacuski, W.; Sobczak, K.; Kazimierczuk, T.; Golnik, A.; Suffczyński, J. Long-distance coupling and energy transfer between exciton states in magnetically controlled microcavities. Communications Materials 2020, 1, 78.

(14) Kasprzak, J.; Richard, M.; Kundermann, S.; Baas, A.; Jeambrun, P.; Keeling, J. M. J.; Marchetti, F. M.; Szymanska, M. H.; Andre, R.; Staehli, J. L.; Savona, V.; Littlewood, P. B.; Deveaud, B.; Dang, L. S.
Bose-Einstein condensation of exciton polaritons. Nature 2006, 443, 409-14.

(15) Klaers, J.; Schmitt, J.; Vewinger, F.; Weitz, M. Bose-Einstein condensation of photons in an optical microcavity. Nature 2010, 468, $545-8$.

(16) Thangaduati, T. D.; Manjubaashini, N.; Thomas, S.; Maria, H. J. Nanostruct. Mater.; Springer, 2020.

(17) McCord, M. A.; Rooks, M. J. Handbook of Microlithography, Micromachining and Microfabrication; SPIE, 2000.

(18) Jaeger, R. C. Introduction to microelectronic fabrication; Prentice Hall, 2002.

(19) Huang, S.-C.; Hong, K.-B.; Chiu, H.-L.; Lan, S.-W.; Chang, T.C.; Li, H.; Lu, T.-C. Design of photonic crystal surface emitting lasers with indium-tin-oxide top claddings. Appl. Phys. Lett. 2018, 112, 061105 .

(20) Chou, S. Y.; Krauss, P. R.; Renstrom, P. J. Imprint Lithography with 25-Nanometer Resolution. Science 1996, 272 (5258), 85-7.

(21) Gates, B. D.; Xu, Q.; Stewart, M.; Ryan, D.; Willson, C. G.; Whitesides, G. M. New Approaches to Nanofabrication: Molding, Printing, and Other Techniques. Chem. Rev. 2005, 105 (4), 11711196.

(22) Lu, Y.; Huang, J. Y.; Wang, C.; Sun, S.; Lou, J. Cold Welding of Ultrathin Gold Nanowires. Nat. Nanotechnol. 2010, 5 (3), 218-224.

(23) Nojiri, K. Dry Etching Technology for Semiconductors; Springer, 2015.

(24) Michalzik, R. VCSELs: Fundamentals, Technology and Applications of Vertical-Cavity Surface-Emitting Lasers; Springer, 2013.

(25) Lott, J. A.; Schneider, R. P.; Malloy, K. J.; Kilcoyne, S. P.; Choquette, K. D. Partial top dielectric stack distributed Bragg reflectors for red vertical cavity surface emitting laser arrays. IEEE Photonics Technol. Lett. 1994, 6 (12), 1397-1399.

(26) Amann, M. Tuning triumph. Nat. Photonics 2008, 2, 134-135.

(27) Dems, M. Modelling of high-contrast grating mirrors. The impact of imperfections on their performance in VCSELs. OptoElectron. Rev. 2011, 19, 340.

(28) Jandura, D.; Czyszanowski, T.; Pudis, D.; Marciniak, M.; Goraus, M.; Urbancova, P. Polymer-based MHCG as selective mirror. Appl. Surf. Sci. 2020, 527, 146827.

(29) Czyszanowski, T.; Sokól, A. K.; Dems, M.; Wasiak, M. Transparent electrode employing deep subwavelength monolithic high-contrast grating integrated with metal. Opt. Express 2020, 28, 28383.

(30) Komar, P.; Gȩbski, M.; Lott, J. A.; Czyszanowski, T.; Wasiak, M. Experimental Demonstration of Light Focusing Enabled by Monolithic High-Contrast Grating Mirrors. ACS Appl. Mater. Interfaces 2021, 13, 25533-25539.

(31) Czyszanowski, T. Quantum-cascade vertical-cavity surfaceemitting laser. IEEE Photonics Technol. Lett. 2018, 30 (4), 351-354.

(32) Totsu, K.; Fujishiro, K.; Tanaka, S.; Esashi, M. Fabrication of three-dimensional microstructure using maskless gray-scale lithography. Sens. Actuators, A 2006, 130, 387-392.

(33) Waits, C. M.; Modafe, A.; Ghodssi, R. Investigation of grayscale technology for large area 3D silicon MEMS structures. $J$. Micromech. and Microeng. 2003, 13 (2), 170.

(34) Holmstrom, T.; Baran, U.; Urey, H. Mems laser scanners: a review. J. Microelectromech. Syst. 2014, 23, 259-275.

(35) Dems, M.; Kotyński, R.; Panajotov, K. Planewave admittance method - a novel approach for determining the electromag-netic modes in photonic structures. Opt. Express 2005, 13, 3196.

(36) Snyder, A.; Love, J. Optical Waveguide Theory; Springer: U.S.A., 1984.

(37) Qiao, P.; Yang, W.; Chang-Hasnain, C. J. Recent advances in high-contrast metastructures, metasurfaces, and photonic crystals. Adv. Opt. Photonics 2018, 10, 180-245. 